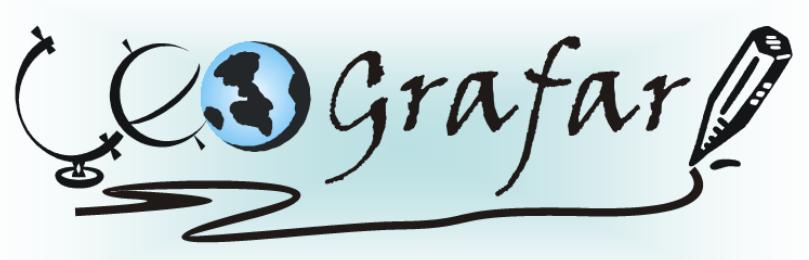

Revista Eletrônica do Programa de Pós-Graduação em Geografia - UFPR

\title{
VARIAÇÃO MENSAL E SAZONAL DA NEBULOSIDADE EM SANTA MARIA, RIO GRANDE DO SUL, NO PERÍODO DE 1969 A 2005.
}

\author{
CÁSSIO ARTHUR WOLLMANN ${ }^{1}$ \\ MARIA DA GRAÇA BARROS SARTORI ${ }^{2}$
}

\begin{abstract}
Resumo: Analisar a variação temporal dos elementos meteorológicos constitui-se em uma das preocupações mais antigas dos estudos em climatologia. No Rio Grande do Sul, os estudos a cerca da variação temporal da nebulosidade restringem-se a poucos trabalhos realizados recentemente. Assim, esta pesquisa pretendeu avaliar a variação mensal e sazonal da nebulosidade no Estado através da duração, em dias, de classes de nebulosidade ao longo do ano. Para tal, utilizou-se o município de Santa Maria/RS como indicador espacial e fez-se fundamentação teórica sobre dinâmica atmosférica regional. Foram utilizados dados climáticos da Estação Meteorológica da UFSM localizada no Município, do período entre 1969 a 2005, totalizando 37 anos de análise. Fez-se, também, a contagem dos dados utilizando-se classes equivalentes de nebulosidade. Para cada três dados pertencentes a uma mesma classe de nebulosidade, marcava-se 1 (um) dia de participação da mesma durante o mês analisado. Ao final, somou-se a quantidade de dias dominados pela mesma classe em cada mês e em cada estação do ano, e se buscou a sazonalidade. Verificou-se que ocorre variação ao longo do ano no número de dias de participação de cada classe de nebulosidade que se dá, principalmente, em função da gênese e deslocamento dos principais sistemas atmosféricos que atuam no Rio Grande do Sul ao longo do ano. Nesse sentido, as classes de nebulosidade de 0 a 3 e de 7 a 10 décimos de céu coberto apresentaram quadros diretamente proporcionais e cíclicos ao longo do ano, aumentando sua participação durante o inverno e diminuindo durante os meses de verão. No entanto, a classe de 4 a 6 décimos de céu coberto apresentou variação anual inversamente proporcional ao das outras duas classes, atingindo seu mínimo nos meses de inverno e máximo de participação nos meses de verão.
\end{abstract}

Palavras-chave: Variação, Nebulosidade, Rio Grande do Sul.

\section{SEASONAL AND MONTH VARIATION OF CLOUDINESS IN SANTA MARIA, RIO GRANDE DO SUL, FROM 1969 TO 2005}

\begin{abstract}
To analyze the temporal variation of meteorological elements constitutes one of the oldest concerns of the studies in climatology. In Rio Grande do Sul, studies about the temporal variations of cloudiness are restricted to few studies carried out recently. Thus, this research sought to evaluate the monthly and seasonal variation of cloud cover in the State through the duration of days of classes of cloudiness over the years. For this, it was used the city of Santa Maria/RS as a spatial indicator and it was done the theoretical foundation on regional atmospheric dynamics. It was used climate data from meteorological station UFSM located in the municipality, the period between 1969 to 2005, totaling 37 years of analysis. Afterwards, it was done the counting of the data using equivalent classes of cloudiness. For every three data belonging to a same class of clouds, it was marked one day of participation of the same day during the months analyzed. In the end, the number of days was summed up, dominated by the same class in each month and each season, and we seek for seasonality. It was found that variation occurs throughout the year in the number of days of
\end{abstract}

\footnotetext{
${ }_{1}^{1}$ Geógrafo/UFSM - Pós-Graduando/USP - cassio_geo@yahoo.com.br

2 Prof ${ }^{2}$. Dra . Departamento o Geografia/UFSM - magracas@base.ufsm.br
} 
participation of each class of cloud that occurs mainly on the basis of the genesis and movement of major weather systems that operate in Rio Grande do Sul throughout the year. In this sense, the classes of cloudiness $0-3$ and 7-10 tenths of sky covered presented situations directly proportional and cyclical over the year, increasing its participation during the winter and decreasing during the summer months. However, the class 4-6 tenths of sky covered showed annual variation inversely proportionall to the other two classes, reaching its minimum in winter and maximum participation in the summer months.

Key-words: Variation; Cloudiness; Rio Grande do Sul.

\section{INTRODUÇÃO}

Analisar a variação temporal dos elementos meteorológicos constitui-se em um dos ramos mais antigos dos estudos em climatologia no Mundo e no Brasil. Avaliar essa variação permitiu, durante muitos anos, conhecerem empiricamente as condições da variabilidade das condições atmosféricas, geralmente, ao longo de um ano sobre determinada região, a fim de se determinar um padrão sazonal.

A variação sazonal da nebulosidade está intrinsecamente ligada à dinâmica atmosférica regional, ou seja, ao deslocamento de massas de ar e correntes perturbadas sobre determinada região. No Brasil Tropical e Equatorial, por exemplo, em especial na fachada atlântica, a variação sazonal da participação dos sistemas atmosféricos é aparentemente homogênea entre as diferentes estações do ano (Nimer, 1979).

No Estado do Rio Grande do Sul, de acordo com Sartori $(1979,1980,1981)$ que se situa em região de transição climática entre as condições extratropicais e intertropicais no que tange a atuação dos sistemas produtores do tempo e do clima, pode também refletir sobre as condições de nebulosidade, uma variação sazonal diretamente ligada à dinâmica atmosférica regional e sazonal (Circulação Secundária).

No Estado, os estudos a cerca da variação temporal da nebulosidade restringem-se aos de Wollmann; Sartori; Ruoso; et. al. (2006a, 2006b), nos quais os autores tratam do percentual de participação de diferentes classes de nebulosidade ao longo do ano, ligando tal fato à percepção da população em relação ao total pluviométrico anual no Rio Grande do Sul.

Dessa forma, a presente pesquisa pretendeu avaliar a variação mensal e sazonal da nebulosidade no Rio Grande do Sul através da duração (em dias) das 
classes de nebulosidade (Wollmann; Sartori; Ruoso; et. al., op. cit.) ao longo do ano, visto que a quase inexistência de tal tipo de trabalho ainda é fato nos estudos de Climatologia Geográfica no Estado.

\section{METODOLOGIA}

Para avaliar o comportamento mensal e sazonal da nebulosidade no Rio Grande do Sul, utilizou-se Santa Maria/RS (Latitude $29^{\circ} 45^{\prime}$ S; Longitude $53^{\circ} 45^{\prime} \mathrm{W}$ ) como indicador espacial da variação da cobertura de nuvens (Figura 1).

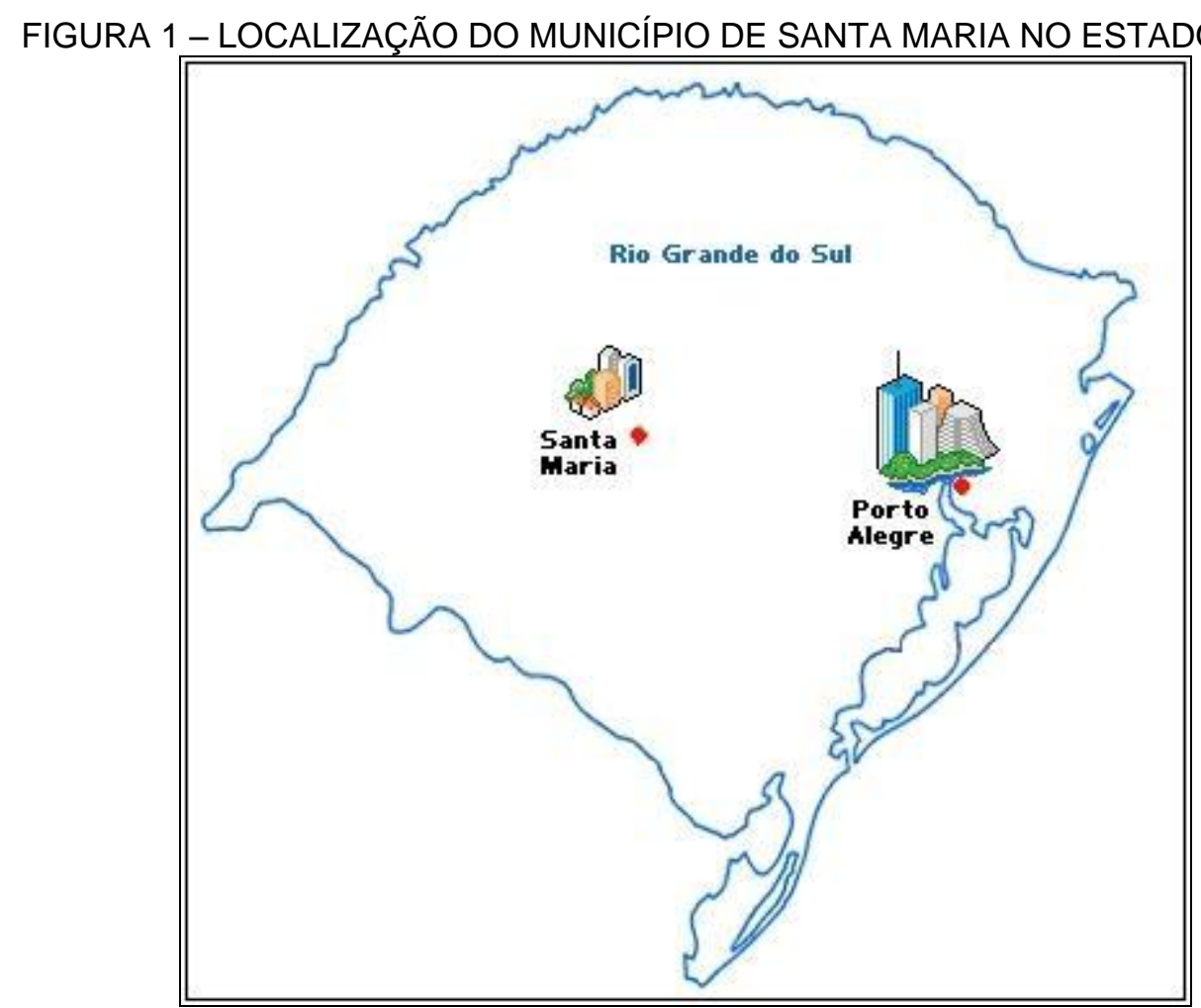

Fez-se fundamentação teórico-metodológica sobre dinâmica atmosférica regional, especialmente sobre o Sul do Brasil, bem como leituras em trabalhos de caráter estático sobre a atmosfera. Foram utilizados dados climáticos, em escala diária de análise, da Estação Meteorológica de Santa Maria, localizada no referido Município, do período entre 1969 a 2005, totalizando 37 anos de análise, como mencionado, em escala diária. 
Para a análise dos dados, menciona-se aqui, o uso do "Quadro de Classes Equivalentes de Nebulosidade" de Wollmann; Sartori; Ruoso; et. al. (op. cit.), na qual os autores estabeleceram três classes de nebulosidade para posterior contagem, conforme esclarece a Tabela 1.

TABELA 1 - CLASSES EQUIVALENTES PARA ESTIMAÇÃO DA PARTICIPAÇÃO MENSAL E SAZONAL DA NEBULOSIDADE

\begin{tabular}{lc}
\hline CEN $^{*}(\mathrm{X} / 10)$ & CVN \\
\hline $0-3$ & Pouca nebulosidade \\
$4-6$ & Parcialmente nublado \\
$7-10$ & Muita nebulosidade \\
Fonte: Estação Meteorológica de Santa Maria/RS \\
Org.: WOLLMANN, C. A., 2007. \\
${ }^{*}$ CEN - Classes Equivalentes de Nebulosidade (0/10 de céu coberto)
\end{tabular}

$\mathrm{Na}$ contagem dos dados foi considerado que cada mês possui de 84 (fevereiro) a 93 registros de nebulosidade (para meses com 31 dias) - (cobertura do céu em décimos), em função de a observação ser realizada três vezes ao dia, nos horários oficiais estabelecidos pela Organização Meteorológica Mundial (OMM).

Assim, para cada três dados contados pertencentes a uma mesma classe de nebulosidade, marcava-se 1 (um) dia de participação da mesma durante o mês analisado. Ao final, somou-se a quantidade de dias dominados pela mesma classe de nebulosidade em cada mês e em cada estação do ano, a fim de se buscar uma sazonalidade na variação da cobertura de céu.

Por fim, os dados foram digitados e tabulados nos aplicativos Microsoft Excel e Word 2003, com montagem de tabelas e gráficos que podem ser visualizados no decorrer do trabalho, a partir dos quais foi possível estabelecer a sazonalidade de participação das classes de nebulosidade.

\section{FUNDAMENTAÇÃO TEÓRICA}

Analisar e entender a variação da nebulosidade sobre algum lugar da superfície terrestre constitui-se numa das linhas de pesquisa da climatologia brasileira. Para tal, TUBELIS; NASCIMENTO (s/d, p. 179) definem a nebulosidade 
como a “... fração do céu que se apresenta coberta por nuvens no momento da observação. É expressa em décimos de céu coberto".

A nebulosidade, assim como todos os outros elementos climáticos, não é regular ao longo de um ano, possuindo sazonalidade. Ainda, para os autores estudados:

\footnotetext{
“... o curso anual da nebulosidade não é regular como o da radiação global, por exemplo, mas depende da época do ano em que determinados processos de formação de nuvens atuam. Uma vez que as precipitações se originam da nebulosidade, os seus cursos anuais são similares... A precipitação é diretamente proporcional à nebulosidade, acompanhando as suas flutuações ao longo do ano" TUBELIS; NASCIMENTO (s/d, p. 179)
}

No Brasil, há poucos estudos sobre a variação temporal da nebulosidade. Mais uma vez, Tubelis; Nascimento (op. cit), dedutivamente, estabeleceram comportamentos anuais da nebulosidade para diferentes Estados brasileiros. Recentemente, esses estudos foram retomados em Mello (2004, p. 216), que ao analisar a nebulosidade no Estado de Santa Catarina, coloca que este tipo de análise climática permite mostrar o importante papel que o estudo das variáveis meteorológicas desempenham no contexto dos estudos realizados pela Climatologia Geográfica brasileira.

Também em ARAÚJO (2006, p. 256), que analisou a variação da nebulosidade na Paraíba e sua influência nas perdas de $\mathrm{C}-\mathrm{CO}_{2}$ no solo do semi-árido, coloca que "A amplitude de variação anual da nebulosidade na região é relativamente pequena, variando de 5 a 8/10 ao longo do ano".

No Rio Grande do Sul, nos primeiros estudos sobre o clima do Estado, Araújo (1930), Machado (1950) e Moreno (1961) dão ênfase, em seus estudos, à insolação e nevoeiros, bem como temperatura e precipitação, não tratando diretamente da nebulosidade.

TUBELIS; NASCIMENTO, (op. cit., p. 181-182), por sua vez, trabalham com a variação mensal dos dados de nebulosidade, colocando que: "Na Região Sul, ocorrem dois máximos, um em setembro e outro em junho [...] e no Rio Grande do Sul uma outra máxima em dezembro", salientando, ainda, que "[...] a menor 
nebulosidade anual é no Rio Grande do Sul.", em comparação com os Estados do Pará, Mato Grosso, São Paulo e Paraíba.

Em Sartori (1981, 1993b, 2003), a nebulosidade é vista como uma característica marcante da gênese do clima do Estado, que se baseia na sucessão de tipos de tempo específicos e condicionados pela circulação atmosférica no Sul do Brasil, especialmente naqueles em que há a participação de correntes perturbadas, tais como as Frentes Polares e Instabilidades Tropicais.

Por último, WOLLMANN; SARTORI; RUOSO; et. al. (2006b) traz um novo enfoque ao estudo da variação anual da nebulosidade no Estado. Para os autores, a nebulosidade varia sazonalmente ao longo do ano, sendo capaz de influenciar na percepção da população sobre o total pluviométrico registrado em função da cobertura de nuvens ser maior em uma estação do que em outra, levando a população a inferir que o volume de chuvas concentrar-se-ia mais no inverno do que nas outras três estações, quando a nebulosidade é menor, ressaltando que:

\begin{abstract}
"Assim, a percepção da população... em relação ao índice de chuvas sazonais não se comprova. No entanto, o fato que pode explicar esse conhecimento empírico é a variação temporal e sazonal da nebulosidade e a duração do dia ao longo do ano no Rio Grande do Sul. Essa variação, de certa forma, altera os processos biológicos e psicológicos do organismo humano, em especial os que ocorrem no cérebro, já que este controla boa parte das funções orgânicas de nosso organismo, bem como a percepção e cognição ambiental" WOLLMANN;SARTORI;RUOSO;et. al. (2006b, p. 910.).
\end{abstract}

Cabe ressaltar que, segundo Sartori (1993a, 1993c), no Rio Grande do Sul não há uma estação definida como chuvosa ou seca, pois as pluviometrias registradas nas quatro estações são percentualmente idênticas, ou seja, as chuvas são regularmente distribuídas ao longo do ano. Há, sim, grande variabilidade da pluviometria mensal e que pode acontecer em qualquer estação do ano, resultantes de distúrbios na circulação secundária regional.

Nesse sentido, WOLLMANN; SARTORI; RUOSO; et. al. (op. cit., p. 8) identificou uma variação anual da nebulosidade, analisada em classes, verificando que os maiores valores de participação são registrados no inverno, afirmando que: 


\begin{abstract}
"Em situação de verão, os sistemas atmosféricos de alta pressão estão mais enfraquecidos pelo aquecimento geral do Hemisfério Sul, e, conseqüentemente, as frontogêneses no Sul do Brasil ocorrem com menor intensidade, não promovendo a formação de grande nebulosidade, tanto em espessura quanto na largura da faixa de nuvens [...] No inverno, quando os sistemas atmosféricos de alta pressão se encontram fortalecidos pelo resfriamento do Hemisfério Sul e pelo aumento do gradiente térmico entre altas latitudes e o Equador, que reforçam o abastecimento polar [...], as frontogêneses se tornam mais intensas no Sul do Brasil, provocando um aumento geral da nebulosidade".
\end{abstract}

Ressalta-se que, ao basear-se nos trabalhos de Sartori (1980: 107-108), os autores colocam que a gênese e a sucessão dos diferentes tipos de tempo ao longo do ano faz com que, no inverno, a nebulosidade se torne maior, em função da maior freqüência de participação de tipos de tempo característicos de passagens frontais, nos quais a nebulosidade alta e persistente torna-se marcante.

Vê-se, portanto, que os resultados encontrados pelo autor, ao serem comparados com os de Tubelis; Nascimento (op. cit.) não possuem conclusões diferentes entre si. Todavia, esses últimos possuem um caráter empírico em relação à medição da nebulosidade, não relacionando com a dinâmica atmosférica particular de cada região, como apresentou Wollmann; Sartori; Ruoso; et. al. (op. cit.) para o Rio Grande do Sul.

\title{
RESULTADOS E DISCUSSÕES
}

Depois de analisados os dados, partiu-se para a construção das tabelas e gráficos que melhor representariam a variação mensal, sazonal e anual de classes de nebulosidade na região central do Rio Grande do Sul. Nesse sentido, a Tabela 2 mostra a variação mensal da nebulosidade em número de dias de participação, classe por classe. 
TABELA 2 - VARIAÇÃO MENSAL DA NEBULOSIDADE EM N DE DIAS DE PARTICIPAÇÃO. PERÍODO DE ANÁLISE: JAN. 1969 - DEZ. 2005.

\begin{tabular}{ccccccccccccc}
\hline $\mathrm{CN}^{*}$ & Jan. & Fev. & Mar. & Abr. & Maio & Jun. & Jul. & Ago. & Set. & Out. & Nov. & Dez. \\
\hline $0-3$ & 8 & 7 & 9 & 9 & 10 & 9 & 10 & 10 & 9 & 9 & 11 & 9 \\
$4-6$ & 7 & 7 & 6 & 5 & 4 & 4 & 4 & 3 & 3 & 4 & 5 & 6 \\
$7-10$ & 16 & 14 & 16 & 16 & 17 & 17 & 17 & 18 & 18 & 18 & 14 & 16 \\
TDM $^{*}$ & 31 & 28 & 31 & 30 & 31 & 30 & 31 & 31 & 30 & 31 & 30 & 31 \\
\hline
\end{tabular}

Fonte: Estação Meteorológica de Santa Maria/RS

Org.: WOLLMANN, C. A., 2007.

${ }^{*} \mathrm{CN}$ - Classes de Nebulosidade (0/10 de céu coberto)

*TDM - Total de Dias que compõem o Mês.

Nota-se na Tabela 2, que a classe de 0 a 3 atinge seus mínimos de participação em janeiro e fevereiro, com 8 e 7 dias respectivamente, e aumentando durante os meses de outono, inverno e primavera, variando entre 9 e 10 dias, atingindo seu máximo no mês de novembro (11 dias).

A segunda classe, com valores entre 4 e 6 décimos de céu coberto, possui maior parcela de participação entre os meses de dezembro e março (verão), sendo que, no restante do ano, a variação atinge no máximo 5 dias e no mínimo 3, correspondendo, respectivamente, aos meses de abril/novembro e agosto/setembro.

A classe representativa de maior nebulosidade, 7 a 10 décimos de céu coberto, apesar de ser a de maior representatividade ao longo do ano, apresenta dois mínimos de participação mensal: um em novembro e outro em fevereiro, ambos os meses com 14 dias de domínio desta classe.

A Tabela 3, por sua vez, mostra a variação sazonal da nebulosidade, também representada em classes equivalentes de nebulosidade. 
TABELA 3 - VARIAÇÃO SAZONAL DA NEBULOSIDADE EM N DE DIAS DE PARTICIPAÇÃO. PERÍODO DE ANÁLISE: JAN. 1969 - DEZ. 2005.

\begin{tabular}{ccccc}
\hline CN $^{*}$ & Primavera & Verão & Outono & Inverno \\
\hline $0-3$ & 29 & 24 & 28 & 29 \\
$4-6$ & 12 & 20 & 15 & 11 \\
$7-10$ & 50 & 46 & 49 & 52 \\
TDE $^{*}$ & 91 & 90 & 92 & 92 \\
\hline
\end{tabular}

Fonte: Estação Meteorológica de Santa Maria/RS

Org.: WOLLMANN, C. A., 2007.

${ }^{*} \mathrm{CN}$ - Classes de Nebulosidade (0/10 de céu coberto)

*TDE - Total de Dias que compõem a Estação do ano.

A Tabela 3 revela que a classe representante da nebulosidade mais baixa entre 0 e 3 possui maior participação, em número de dias, no inverno e na primavera, ambas estações com 29 dias, reduzindo sua participação em número de dias no outono e no verão.

A segunda classe, ou seja, de 4 a 6 décimos de céu coberto, é a que apresenta a maior variação sazonal dentre três analisadas, partindo desde 11 dias no inverno até 20 dias no verão, permanecendo a primavera e o outono como estações de transição, com 12 e 15 dias de participação, respectivamente.

A classe de 7 a 10 apresenta seu máximo no inverno e na primavera, com 52 e 50 dias cada, sendo ao verão, a época do ano na qual os maiores índices de nebulosidade registrados são os menos representativos. O outono, por fim, apresenta valores semelhantes ao da primavera, com 49 dias de participação.

Na Tabela 4, observa-se a participação anual, em dias, de domínio, das 3 classes equivalentes de nebulosidade.

TABELA 4 - VARIAÇÃO ANUAL DA NEBULOSIDADE EM N DE DIAS DE PARTICIPAÇÃO.

PERÍODO DE ANÁLISE: JAN. 1969 - DEZ. 2005.

\begin{tabular}{ccc}
\hline CN $^{*}$ & Dias de participação & \% de participação \\
\hline $0-3$ & 110 & 30,1 \\
$4-6$ & 58 & 15,9 \\
$7-10$ & 197 & 54,0 \\
TDA $^{*}$ & 365 & 100 \\
\hline
\end{tabular}

Fonte: Estação Meteorológica de Santa Maria/RS

Org.: WOLLMANN, C. A., 2007.

${ }^{*} \mathrm{CN}$ - Classes de Nebulosidade (0/10 de céu coberto)

*TDA - Total de Dias que compõem o Ano. 
A classe de nebulosidade entre 7 e 10 décimos de céu coberto é a que possui a maior representatividade ao longo do ano, com 54\% de participação, correspondendo a 197 dias do ano. Em segunda colocação, vê-se a classe de menor nebulosidade, 0 a 3 décimos de céu coberto, com 110 dias do ano dominados pela mesma, correspondendo a $30,1 \%$ do total anual. Por último, portanto, a segunda classe de nebulosidade (4 a 6/10) é a que menos participa anualmente: 58 dias, totalizando $15,9 \%$ do total anual das três classes equivalente de nebulosidade.

Na Figura 2 observa-se melhor a variação anual da nebulosidade segundo as três classes de representatividade, em que se constata que a variabilidade mais expressiva é a de 4 a 6 décimos de céu coberto, permanecendo a classe de 0 a 3 e 7 a 10 como as mais homogêneas ao longo do ano, apresentando, ambas, seus mínimos apenas nos meses de verão.

FIGURA 2 - GRÁFICO DE VARIAÇÃO ANUAL DAS CLASSES EQUIVALENTES DE NEBULOSIDADE EM № DE DIAS DE PARTICIPAÇÃO. PERÍODO DE ANÁLISE: JAN. 1969 - DEZ. 2005.

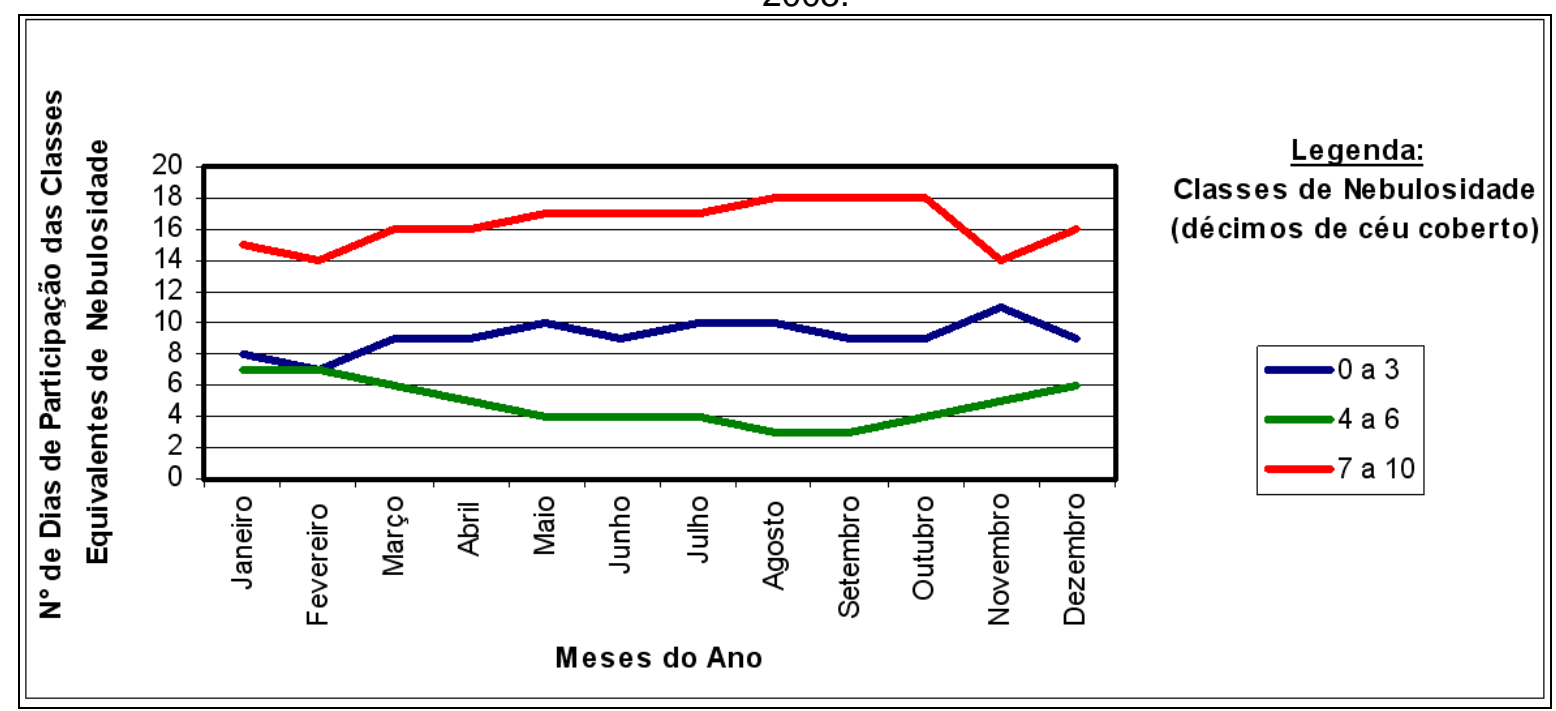

Org.: WOLLMANN, C. A., 2007.

Essa variação ao longo do ano no número de dias de participação de cada classe de nebulosidade se dá, principalmente, em função da gênese e deslocamento dos principais sistemas atmosféricos que atuam no Rio Grande do Sul ao longo do ano.

No verão, por exemplo, as Frentes Polares, que são as principais responsáveis pela formação de nuvens na latitude sul-rio-grandense, seguido das Instabilidades 
Tropicais (Sartori, 2003), em função de um maior aquecimento do Hemisfério Sul como um todo por causa da maior entrada de radiação solar global, leva à desconfiguração das características físicas, tais como a temperatura, pressão e umidade, das massas de ar em confronto no Estado. Nesse sentido, as frontogêneses ocorrem com menor intensidade, logo as classes de nebulosidade extremas (de 0 a 3 e de 7 a 10 décimos de céu coberto) registraram seus mínimos nesta estação.

Em situação climática oposta, ou seja, no inverno, o abastecimento de ar polar nos Anticiclones Migratórios Polares torna-se maior, resultando em frontogêneses intensas, elevando os níveis de nebulosidade e refletindo-se no aumento da participação classes extremas. Nesse sentido, cabe às Classes Equivalentes de Nebulosidade de 0 a 3 e de 7 a 10 décimos de céu coberto, mais uma vez, apresentar uma variação diretamente proporcional e cíclica ao longo do ano, ou seja, aumentam sua participação durante o inverno e diminuem o número de dias de influência durante os meses de verão.

No entanto, com a classe de 4 a 6 décimos de céu coberto, que pode ser considerada uma classe de nebulosidade transicional entre as condições de céu limpo e céu encoberto, apresenta uma variação anual inversamente proporcional ao das outras duas classes, atingindo seu mínimo nos meses de inverno e máximo de participação nos meses de verão.

De posse destes resultados, portanto, ressalta-se que o número de dias de nebulosidade da segunda classe (4 a 6) dá lugar à participação das condições de nebulosidade extrema (pouca e muita nebulosidade) durante o inverno, ocorrendo o oposto durante o verão do Rio Grande do Sul, evidenciando um jogo de forças equilibrado entre os sistemas atmosféricos produtores do tempo, ainda pouco estudado pela climatologia brasileira e mundial. 


\section{CONCLUSÕES}

Neste trabalho ficou evidenciada a variabilidade temporal da nebulosidade no Rio Grande do Sul, expressa em classes equivalentes.

De acordo com os resultados encontrados em Tubelis; Nascimento (op. cit.) foi encontrado dois máximos para o Estado: um em junho e outro em setembro. Ao relacioná-los com os resultados obtidos nesta pesquisa (Tabela 1), vê-se que os máximos da classe de maior nebulosidade realmente ocorrem em junho, setembro e outubro, com 17 e 18 dias de participação respectivamente, comprovando, portanto, que o inverno e a primavera são as estações do ano que apresentam maior cobertura de nuvens (Tabela 2).

Em relação às mínimas encontradas nesta pesquisa (Tabela 1), dezembro não concentrou nenhuma mínima para qualquer uma das três classes de nebulosidade, mas para a mesma classe de 7 a 10 décimos de céu coberto, as mínimas foram registradas em novembro e fevereiro (verão).

Ao final, salienta-se que essa pesquisa teve caráter pioneiro na abordagem dada ao se tratar da variabilidade temporal da nebulosidade no Rio Grande do Sul, visto que se trata de um elemento meteorológico também indispensável na análise do ciclo climático de uma região.

Nesse sentido, sugere-se complementação deste trabalho a um que trate da variação têmporo-espacial da nebulosidade no Estado. Para tal, a obtenção de dados, em escala diária, das diversas regiões torna-se é difícil, pois as Estações Meteorológicas, bem como $08^{\circ}$ Distrito de Meteorologia de Porto Alegre, não armazenam ou disponibilizam gratuitamente os dados em escala diária, imprescindíveis para as pesquisas em Climatologia Geográfica no Brasil atualmente.

\section{REFERÊNCIAS BIBLIOGRÁFICAS}

ARAÚJO, K. D.; ANDRADE, A. P.; ROSA, P. R. O.; PAZERA JR, E. Variação da nebulosidade e sua influência sobre as perdas de $\mathrm{C}-\mathrm{CO}_{2}$ do solo no semi-árido da Paraíba. In: SIMPÓSIO BRASILEIRO DE CLIMATOLOGIA GEOGRÁFICA, 7. Rondonópolis: UFMT, 20-25 ago. 2006a. Anais... 1 CD-ROM. 
MELLO, M. L. Variabilidade temporal e distribuição espacial de brilho solar associada à nebulosidade e precipitação, em Santa Catarina, no período 1981-2001. In: SIMPÓSIO BARSILEIRO DE CLIMATOLOGIA GEOGRÁFICA, 6. Aracaju: UFS, 2004. Anais... 1 CD-ROM.

NIMER, E. Climatologia do Brasil. Rio de Janeiro: IBGE/SUPREN, 1979. p. 9-19.

SARTORI, M. G. B. A dinâmica do clima do Rio Grande do Sul: indução empírica e conhecimento científico. Revista Terra Livre, São Paulo, v. 1, n. 20, p. 27-49, jan./jul. 2003.

SARTORI, M. G. B. Distribuição das chuvas no Rio Grande do Sul e a variabilidade têmporo-espacial no período 1912-1984. In: SMPÓSIO BRASILEIRO DE GEOGRAFIA FÍSICA E APLICADA. 5. São Paulo: USP, Anais... 1993a. p. 275-280.

SARTORI, M. G. B. A circulação atmosférica regional e os principais tipos de sucessão do tempo no inverno do Rio Grande do Sul, Brasil. Ciência e Natura, n. 15, p. 69-93, 1993b.

SARTORI, M. G. B. As variações pluviométricas e o regime das chuvas na região central do Rio Grande do Sul. Boletim de Geografia Teorética. n. 23. p. 70-84. 1993c.

SARTORI, M. G. B. A circulação atmosférica regional e as famílias de tipos de tempo identificadas na região central do Rio Grande do Sul. Ciência e Natura, n. 3, p. 101$110,1981$.

SARTORI, M. G. B. Balanço sazonário da participação dos sistemas atmosféricos em 1973, na região de Santa Maria, RS. Ciência e Natura, n. 2, p. 41-53, 1980.

SARTORI, M. G. B. O clima de Santa Maria: do regional ao urbano. 1979. 163f. Dissertação (Mestrado em Geografia) - Universidade de São Paulo, São Paulo, 1979.

TUBELIS, A. e NASCIMENTO F. J. L. Meteorologia descritiva: Fundamentos e aplicações brasileiras. São Paulo: Nobel, s/d.

WOLLMANN, C. A.; SARTORI, M. G. B.; RUOSO, D.; COSTA, E. R. A influência da nebulosidade na percepção da pluviometria anual no Rio Grande do Sul: Uma relação bioclimática. In: SIMPÓSIO BRASILEIRO DE CLIMATOLOGIA GEOGRÁFICA, 7. Rondonópolis: UFMT, 20-25 ago. 2006a. Anais... 1 CD-ROM.

WOLLMANN, C. A.; SARTORI, M. G. B.; RUOSO, D.; COSTA, E. R.; KLEINPAUL, J. J. A nebulosidade como fator condicionante à percepção da pluviometria anual pela população rural e urbana de São Sebastião do Caí/RS. In: SEMINÁRIO LATINOAMERICANO DE GEOGRAFIA FÍSICA, 4. Maringá: UEM, 14-18 out. 2006b. Anais... 1 CD-ROM. 